\title{
Performance Evaluation of Routing Metrics in the LOADng Routing Protocol
}

\author{
José V. V. Sobral, Joel J. P. C. Rodrigues, Senior \\ Member, IEEE, Neeraj Kumar, Member, IEEE, Chunsheng Zhu, and Raja W. Ahmad
}

\begin{abstract}
LOADng (Lightweight On-demand Ad hoc Distance-vector Routing Protocol - Next Generation) is an emerging routing protocol that emerged as an alternative to RPL (IPv6 Routing Protocol for Low power and Lossy Networks). Although some work has been dedicated to study LOADng, these works do not analyze the performance of this protocol with different routing metrics. A routing metric is responsible for defining values for paths during the route creation process. Moreover, based on these metrics information a routing protocol will select the path to forward a message. Thus, this work aims to realize a performance assessment study considering different routing metrics applied to LOADng. The scenarios under study consider different traffic patterns and network sizes. The routing metrics are evaluated considering the packet delivery ratio, average energy spent per bit delivered, average latency, and number of hops. The results reveals that routing metrics used by this protocol may influence (directly) the network performance.
\end{abstract}

Index Terms-Internet of Things, LOADng, Low power networks, Performance, Routing metric, Routing protocol.

\section{INTRODUCTION}

$\mathbf{T}$ HE concept of Internet of Things (IoT) have emerged with the growing of physical objects connected to the Internet. Predictive studies reveal that the number of interconnected objects through IoT can reach 26 billion until 2020 [1]. The IoT application field is very broad and can cover the sector of industrial manufacturing, energy, transport, e-health, smart cities, agriculture, among others.

Manuscript received February, 25, 2017; revised May, 11, 2017. Date of publication June 1, 2017.

This work has been partially supported by Conselho Nacional de Desenvolvimento Científico e Tecnológico $(\mathrm{CNPq})$ - Brazil through the grant 201155/2015-0, by Instituto de Telecomunicações, Next Generation Networks and Applications Group (NetGNA), Covilhã, Portugal, by National Funding from the FCT - Fundação para a Ciência e a Tecnologia through the UID/EEA/500008/2013 Project, and by Finep, with resources from Funttel, Grant No. 01.14.0231.00, under the Centro de Referência em Radiocomunicações - CRR project of the Instituto Nacional de Telecomunicações (Inatel), Brazil

José V. V. Sobral is with the Instituto de Telecomunicações, Universidade da Beira Interior, Portugal and Federal Institute of Maranhão (IFMA), Maranhão, Brazil (e-mail: jose.sobral@it.ubi.pt)

Joel J. P. C. Rodrigues is with the National Institute of Telecommunications (Inatel), Brazil; Instituto de Telecomunicações, Universidade da Beira Interior, Portugal; and University of Fortaleza (UNIFOR), Ceará, Brazil (e-mail: joeljr@ieee.org)

Neeraj Kumar is with the Thapar University, Patiala (Punjab), India (e-mail: neeraj.kumar@thapar.edu)

Chunsheng Zhu is with the The University of British Columbia, Vancouver, Canada (e-mail: chunsheng.tom.zhu@gmail.com)

Raja W. Ahmad is with the University of Malaya, Kaula Lumpur (e-mail: wasimraja@ ciit.net.pk)

Digital Object Identifier (DOI): 10.24138/jcomss.v13i2.376
A great set of IoT applications is composed by devices with strict restrictions on energy, processing, and bandwidth. These devices exchange data using wireless communication and create a particular type of network called Low Power and Lossy Networks (LLN). Aiming the use of Internet IPv6 on these kind of networks, the Internet Engineering Task Force (IETF) presented several RFCs (Request for Comments) documents. One of these documents defines the RPL protocol (IPv6 Routing Protocol for Low power and Lossy Networks). Proposed in August 2009, RPL was defined as the standard routing protocol for 6LoWPAN (IPv6 over Low power Wireless Personal Area Networks), in March 2012 [2]. From this date, it is common to consider RPL as the standard routing solution for IoT [3]. Although defined as a standard, different studies have exposed that RPL presents some drawbacks [4], [5]. Thus, considering the existing RPL limitations, novel routing protocols have been proposed. Among these new emerging routing solutions, it is possible to detach the LOADng (Lightweight On-demand Ad hoc Distance-vector Routing Protocol - Next Generation) [6], which is a reactive routing protocol designed for LLNs based on the well-know AODV (Ad hoc On-demand Distance Vector routing) [7].

Since the emergence of LOADng as an alternative to RPL, several works have exposed performance assessment studies comparing the two approaches. In [8] the authors compare RPL and LOADng in a home automation scenario with different traffic patterns. In [9] the authors perform the comparison between a reactive (LOADng) and a proactive (RPL) routing protocols in different LLN network topologies. The two protocols are also studied and compared in [10]. Studies considering just LOADng are presented in [11] and [12].

The aforementioned performance studies about LOADng present limitations because they only analyze the default version of the protocol. However, the performance of a routing protocol is influenced by the used routing metrics [13]. A routing metric defines how a routing protocol must compute the weight of each path and select the best route. Thus, based on the necessity of understanding the real potential of the protocol, this work present a performance evaluation study that considers the use of different routing metrics on LOADng. This work differs from the presented in [12] by considering a high variety of routing metrics detaching its importance in the routing performance improvement.

The rest of the document is organized as follows. Section 
II presents the features and operation of LOADng. Section III describes the routing metrics considered in this work while the considered simulation scenarios and networks configurations are addressed at Section IV. The obtained results for each routing metric on different networks are detailed in Section V and Section VI concludes the paper and suggests future works.

\section{LOADNG Routing PROTOCOL}

The LOADng is a reactive routing protocol based on AODV that can be used on Low Power and Lossy Networks (LLNs). Similar to AODV, a route is only created by LOADng when two nodes need to exchange a data message between them. Thus, the control messages perform the route creation [14]. Under this process, a set of information stored by nodes during the protocol operation is also used. Then, the following subsections present the LOADng control messages, its information base, and its operation considering the latest version of the protocol presented in [6].

LOADng uses four control messages: Route Request (RREQ), Route Reply (RREP), Route Reply Acknowledgment (RREP_ACK), and Route Error (RERR). RREQ message is always used when a node needs to send a data message to a destination. RREP is used by a destination node that receives an RREQ as an answer to the route request. RREP_ACK is used to answer an RREP message when it requires an acknowledgment. RRER is used when a node fails at the moment of forwarding a data message to the next hop. RERR can also be used when the destination node of a data message is not known by the forwarding node.

Each node that uses LOADng must maintain an Information Base for controlling the routing processes and the information about the other network nodes. The main elements of the Information Base are the Routing Set, Blacklisted Neighbor Set, and Pending Acknowledgment Set. The Routing Set is composed by a set of routing tuples that stores data about the neighbor nodes, such as the next hop and the number of hops for reaching a destination. Moreover, the valid time of the tuple is stored in the routing set. A routing tuple must be specified as invalid or removed from the Routing Set after its valid time expires. The Blacklisted Neighbor Set stores the nodes address with possible faults that communication has not been possible. Generally, it stores the nodes address that are not able to deliver a required acknowledgment message in a sequence of a communication fault. Each stored address has a valid time that indicates when the information should expire. The Pending Acknowledgment Set records information about the RREP messages that were sent and requires an acknowledgment message (RREP_ACK). Each tuple of the set contains information about the next hop of the RREP, its originator, a sequence number, a flag for indicating if the RREP_ACK was received, and a valid time. If the valid time expires and the RREP_ACK was not received yet, the address of the next hop should be inserted in the Blacklisted Neighbor.

In the LOADng operation, when a node needs to send a data message to a destination, it creates an RREQ message with the same destination of the data message. After creating the RREQ, the node broadcasts the message to all its neighbors.
The RREQ is forwarded until reaching its destination (Figure 1a). When the RREQ destination node is reached, an RREP message is created to answer the originator of the RREQ. The created RREP is sent in unicast, hop by hop, using the information recorded at the Routing Set at the moment of RREQ broadcasts. If necessary, the node that receives an RREP can send an RREP_ACK message to the RREP originator (Figure $1 b$ ). When the RREP reaches its destination, the node that generated the RREQ will be able to send the data packets using the newly created route (Figure 1c).

Although it may seems simple, the processing of RREQ and RREP messages is composed by a set of verifications that seeks to ensure a good protocol operation avoiding loops. Thus, Figure 2 presents a flowchart that shows as a node processes these messages. After receiving an RREQ/RREP message, first, the node verifies whether it is valid for processing. Among others, the validation process verifies if the length of the address (in the message) is different from the length of the receiver node and if the sequence number of the message is lower than the previously received by the message originator. If one of these conditions is true, the message must be dropped. If the message is valid, the used routing metric is computed and updated. Following, the node verifies the existence of a routing tuple for the message originator inside the Routing Set. If a matching tuple is not found, a new routing tuple is created and inserted in the Routing Set. In the next step, the created or found routing tuple is compared with some field of the message to verify if the tuple will be refreshed and if the message will be considered for forwarding. If the expected conditions are not attended, the message is discarded and it is neither considered for forwarding nor refreshing. However, if the conditions are attended, the fields of the message and the refreshed routing metric are used for refreshing the routing tuple. In the next step, the nodes should verify the type of message. If it is an RREQ message, the node verifies if it is the destination. If true, a new RREP message is generated with the originator of the RREQ as a destination. Otherwise, the message is forwarded. On the other hand, if the message is an RREP, the node must verify if an acknowledgment message is required. Then, if true, an RREP_ACK message must be sent to the previous hop of RREP message. Finally, the node verifies if it is the destination of the RREP message. If yes, the process of route creation is finished and the data packets are able to be sent. If not, the message is forwarded to the next hop.

It is also important to note that, for each message received, the field of hop count is incremented and the field of hop limit is decremented. Optionally, if another metric type is used, the field with the value of routing metric may also be updated. Thus, all forwarded messages must be sent with these updated values. It is important to mention that a RREP_ACK message is sent in unicast and cannot be forwarded. A node that receives a RREP_ACK processes it and, after, it is discarded.

LOADng routing protocol is fully based on the AODV. However, some aspects are simplified in order to reduce the protocol complexity and the quantity of computational resources required to execute it. One of the main features refers that only the destination node of an RREQ can answer 


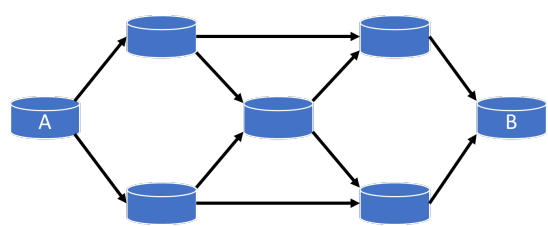

(a) RREQ transmission

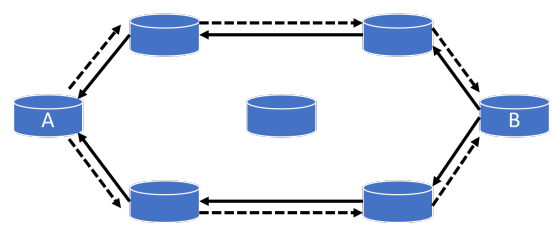

(b) RREP and RREP_ACK (dashed line) transmission

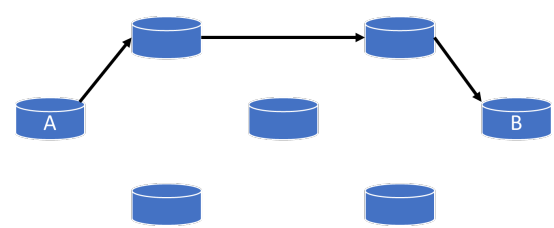

(c) Data transmission

Fig. 1: LOADng operation and use of control messages.

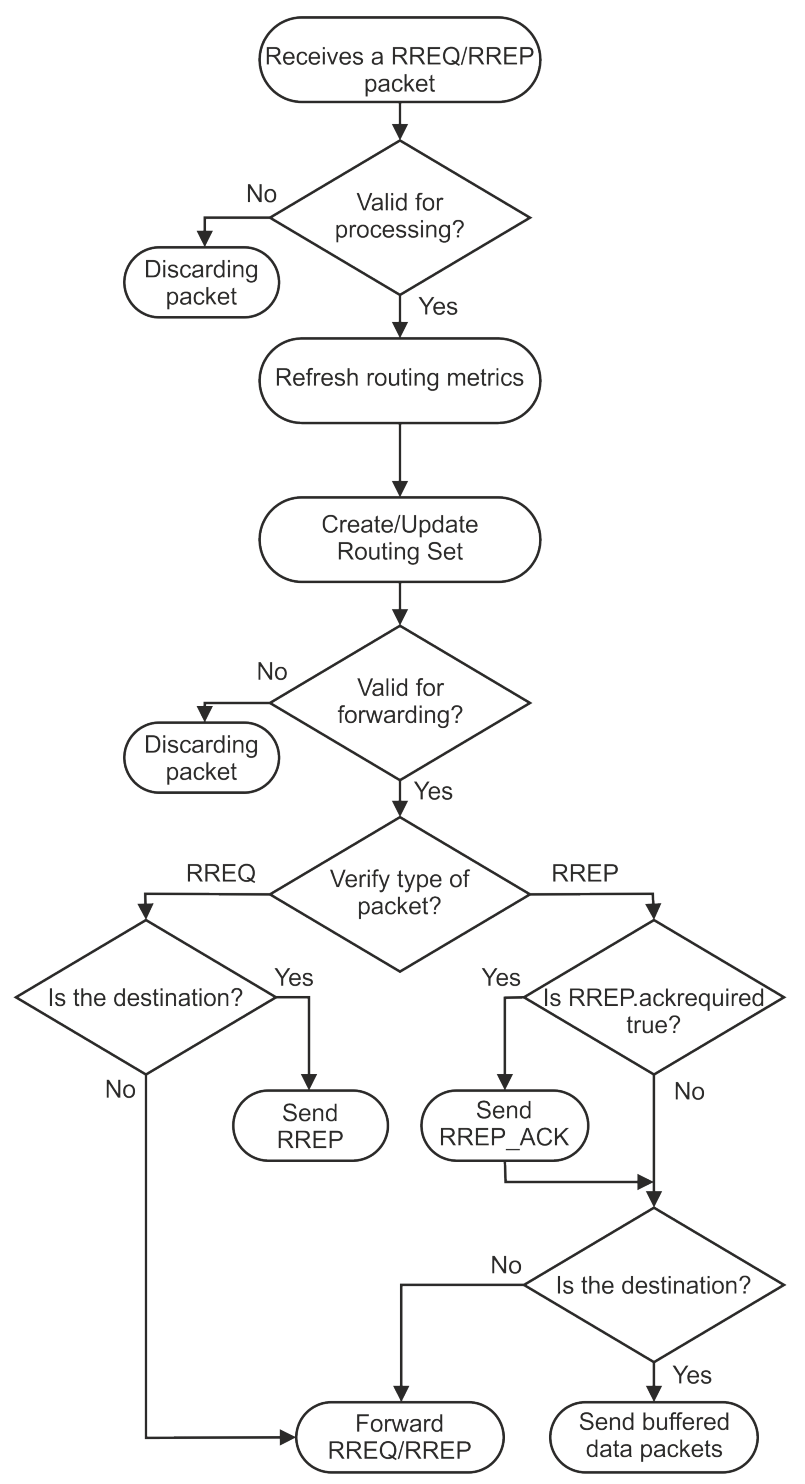

Fig. 2: Illustration of the LOADng Flowchart.

the request with an RREP message. In addition, the nodes do not maintain a list with the address of precursor nodes. In summary, among other features of LOADng, it is possible to highlight the following:

- It supports different lengths of addresses (e.g. IPv6 or IPv4);

- It does not use periodical control messages (e.g., HELLO messages of the AODV);
- It supports the use of different routing metrics optionally to the default hop count.

The possibility of using alternative routing metrics in the LOADng is the primary focus of this work. In the next Section some alternatives to the default hop count that can be used to enhance the routing performance are described.

\section{Routing Metrics}

By default, the LOADng protocol uses the hop count metric for selecting the path (the shortest path, in this case) between two nodes. However, as above-mentioned, it is possible to use different information for computing the weight of the routes. The performance of a routing protocol is strongly related to the routing metric under use. During the route creation process (transmission of RREQs and RREPs), the LOADng uses information of the control messages or calculated at the moment of the signal received for computing the weight of each path according to the routing metric. The calculated values are used to update the routing table with the best route to a destination. After that, these values are forwarded to the other nodes inside of control messages. Thus, the routing table stores the best route to a destination based on the routing metric information.

The available routing metrics are extremely diverse and are categorized into two types: node metrics and link metrics [15]. Node metrics consider aspects related to the node, as processing capacity, remaining energy, etc. On the other hand, the link metrics address information about the connection among nodes, such as delay, link quality, and throughput [16]. The following subsections introduce different link and node metrics that are used with LOADng in this work.

\section{A. Hop Count}

The Hop Count (HC) metric is one of the most commonly used routing metrics. It is the default routing metric of LOADng. HC represents the number of times that a message was sent until reach the destination [17]. Each link in a route counts as one unity without considering neither node nor link characteristics. In an "optimal" scenario (without interference, noises, collision, and energy restrictions), HC can represent the best path due to the use of a small number of transmissions resulting in a low latency and low energy consumption. However, in a real scenario, sometimes, the shortest path may not be the best. Wireless communications commonly suffer from noises and interference that can reduce the link quality among nodes causing packets loss. Thus, the 


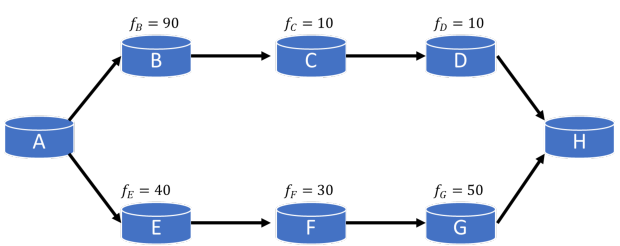

Fig. 3: Example of network composed by nodes with different remaining energies.

HC can not represent the best route since does not consider node nor link quality aspects.

\section{B. Minimum Battery Cost Routing (MBCR)}

The Minimum Battery Cost Routing (MBCR) is a routing metric based on the nodes energy. The MBCR considers the remaining battery capacity of the node for calculating the best path between two nodes [18]. The strategy of the MBCR is to avoid the routes with low remaining energy aiming to reduce the packet loss and decrease the power consumption of the overall network. Thus, the cost of each node $n$ that composes a route $r$ is computed by $f_{n}\left(E_{n}\right)$, where $f_{n}$ is a battery cost function and $E_{n}$ is the current remaining node battery. The $f_{n}$ can be computed using Equation 1.

$$
f_{n}\left(E_{n}\right)=\frac{1}{E_{n}}
$$

The total cost of a route $r$ is computed with the sum of all $f_{n}\left(E_{n}\right)$ (Equation 2). As the MBCR is a minimized metric, the routing protocol should select the route with the minimum total cost.

$$
\sum_{n \in r} f_{n}\left(E_{n}\right)
$$

Although MBCR may provide a network load balancing, it can select routes with low remaining energy nodes. Figure 3 exposes an example where the route A-B-C-D-H is chosen because it presents the lower total sum. However, node B has a very low remaining battery and probably should break the route rapidly.

\section{Min-Max Battery Cost Routing}

Similar to MBCR, the Min-Max Battery Cost Routing (MMBCR) is also a routing metric based on the node energy. However, to solve the main fault of MBCR, the idea of MMBCR is to avoid the use of a route when the nodes have low remaining battery.

Considering that high values obtained with $f_{n}\left(E_{n}\right)$ represent a low remaining node battery, the idea of MMBCR is to choose the path with the minimum function cost. Different to MBCR, the cost of each path is represented by the maximum value of $f_{n}\left(E_{n}\right)$ among the nodes that compose a route. Thus, Equation 3 represents the function to obtain the best path $r^{\prime}$.

$$
r^{\prime}=\min _{r \in R}\left(\max _{n \in r}\left(f_{n}\left(E_{n}\right)\right)\right)
$$

For example, considering the routes of Figure 3, the cost of route A-B-C-D-H computed by MMBCR is 90 (node B) because it is the higher $f_{n}\left(E_{n}\right)$ value along the path. Likewise, the cost of route A-E-F-G-H defined by MMBCR is 50 (node $\mathrm{G})$. To choose the best route $\left(r^{\prime}\right)$, MMBCR gets the path with the minimum cost among those available ones, in this case, the route A-E-F-G-H.

The approach presented by MMBCR may avoid the use of routes composed by nodes with low remaining energy and increase the network lifetime (when the lifetime of the first dead node is considered as the network lifetime). However, the use of MMBCR may not ensure a good network performance. Since that does not take into account information about the link quality among nodes, the selected best route may have nodes with communication faults provoking the packet loss.

\section{LQI Weaklinks}

The Link Quality Indicator Weaklinks (LQI_WL) is a routing metric based on the quality of communication among nodes. The LQI is a real value provided by the physical layer of the standard IEEE 802.15.4. This value, which ranging between 0 (worst) and 255 (best), is computed by a node every time that it receives a message. The calculated value is highly dynamic and may change due to several factors. Thus, the LQI value computed at the transmission $A \rightarrow B$ almost never is equal to the value calculated at the transmission $B \rightarrow A$.

The LQI represents the quality of communication between two nodes (point-to-point). Thus, using the LQI to measure the quality of an end-to-end route, the LQI_WL uses the WeakLinks role. The WeakLinks approach uses a threshold to distinguish links between bad or good. During the route creation process, each node verifies if the computed LQI value is lower than the previously defined threshold $\left(L Q I_{t h}\right)$. If positive, the WeakLinks counter is increased by 1 , else the WeakLinks counter does not change. In this way, the best route between a sender and a destination node is the one with the lower number of WeakLinks among those available ones.

\section{E. $M A X-L Q I$}

The MAX-LQI is a routing metric based on LQI, such as LQI_WL. Working like MMBCR, the MAX-LQI aims to choice the path with the best worst link. Thus, the worst LQI value of the links that composes a route represents the path cost. The routing protocol must select the path with the best cost (maximum LQI) among those available ones.

Since it considers just the worst LQI value of a path, MAXLQI may not select a route with a low number of hops. As a consequence, the number of transmissions used to deliver a message to its destination may be high and the spent energy increased [19].

\section{F. ETX}

The Expected Transmission Count (ETX) [20] is one of the most used routing metrics in LLNs. With a cross-layer approach, ETX determines the expected number of transmissions that a node should perform for the message in order 
to reach its destination successfully. To compute ETX, the nodes store information about the MAC layer packets sent to their neighbors and the acknowledgment packets received. Therefore, it is possible to calculate the number $s$ of packets sent successfully and the number $f$ of packets lost (without receiving acknowledgment). Thus, Equation 4 presents the ETX computation of the link between the nodes $i$ and $j$ [13]

$$
E T X_{i, j}=\frac{s+f}{s}>0
$$

Similar to LQI, ETX defines a weight for a link where the ETX of $A \rightarrow B$ may not be equals to the ETX of $B \rightarrow A$. Thus, the total cost of a path is represented by the sum of all ETX values of the links that compose the route.

The ETX is a minimized routing metric that, for being additive, considers the number of hop among the nodes implicitly. Hence, the use of ETX allows the routing protocol to select a short path composed by reliable links. In contrast, the information stored by nodes to compute the ETX value of its neighbors may exhaust its limited memory resource and restrict the network scalability.

\section{Performance Evaluation}

This work aims to evaluate the performance of primary routing metrics applied to the LOADng routing protocol. The performance assessment study was conducted through simulation using Castalia [21]. Simulations considering two IoT applications with MP2P and $\mathrm{P} 2 \mathrm{P}$ traffic pattern were performed. The MP2P traffic pattern is characterized by the network data traffic flows from the nodes to a central unit (sink or gateway). On the other hand, in $\mathrm{P} 2 \mathrm{P}$ traffic pattern, the network data traffic is directly exchanged among nodes [3].

In the simulated MP2P application, a sink node was placed at the center of the simulation area for receiving messages sent from the other network nodes. In the P2P application, just one node sent messages to a receiver node. The sender was located at the bottom-right of the network, and the receiver was placed at the top-left of the network. In both applications the nodes were static. Table I exposes the simulations parameters used in this study.

The performance assessment study was conducted considering four evaluation metrics: packet delivery ratio, average latency, average spent energy per bit, and number of hops. These metrics are described as follows:

- Packet delivery ratio: represents the quantity of data messages that were delivered to their destination node successfully. A low delivery ratio exposes a fault network efficiency and a limited quality of service. The used routing metric can affect the packet delivery ratio directly since the forwarded messages through routes composed by links with low quality may cause packet loss.

- Average spent energy per bit: represents the amount of energy that network consumes to deliver each data bit successfully. The metric value is obtained through the ratio of the amount of spent energy by the network and the quantity of data bits received by the nodes. Great
TABLE I: Simulation parameters.

\begin{tabular}{|c|c|}
\hline \multicolumn{2}{|c|}{ Simulation parameters } \\
\hline Parameter & Value \\
\hline SimTime & $600 \mathrm{~s}$ \\
\hline Initial Energy & $20 \mathrm{~J}$ \\
\hline Application & MP2P and P2P \\
\hline Routing & LOADng \\
\hline Mac Protocol & 802.15 .4 \\
\hline Radio & CC2420 \\
\hline Data message rate & $0.5 \mathrm{msg} / \mathrm{s}$ \\
\hline Numbe of nodes & $16,36,64$ \\
\hline Simulation area $\left(\mathrm{m}^{2}\right)$ & $50,50,100$ \\
\hline Network deployment & $4 \times 4,6 \times 6,8 \times 8$ grids \\
\hline \multicolumn{2}{|c|}{ Packet length } \\
\hline Type of packet & Lenght \\
\hline RREQ & 240 bits \\
\hline RREP & 272 bits \\
\hline RERR & 240 bits \\
\hline RREP_ACK & 144 bits \\
\hline Data Packet Size & $512+64$ (overhead) bits \\
\hline
\end{tabular}

values of the averaged spent energy per bit show that network is using a high amount of energy to deliver few data messages. Thus, the routing metric should allow that routing protocol optimizes the route selection process to ensure a high packet delivery ratio with an efficient power consumption.

- Average latency: measures the time spent by the network to deliver a data message to its destination. Several aspects can contribute for a low latency such as the physical distance between the sender and the destination node, the nodes workload, the quality of the links, etc. The routing protocol, based on the used routing metric, should avoid paths with a high number of hops and low link quality for trying to ensure an acceptable average latency able to attend the application requirements.

- Number of hops: exposes the number of times that a data message was forwarded until reaching its destination. A high number of hops implies a significant number of the message forwarding and, consequently, a high energy consumption. Hence, it is important that the routing protocol uses short paths but without ignoring the others route aspects as link quality and nodes energy.

Based on the performed experiments using the abovedescribed scenarios and corresponding parameters, next section is dedicated to the results analyses obtained for the four evaluation metrics.

\section{Results AnAlysis}

\section{A. MP2P Application}

Figure 4 depicts the results obtained for the packet delivery ratio metric. In the small network (16 nodes), all the routing metrics can deliver around $90 \%$ of the sent packets. However, with the network growth, the routing metrics have a considerable performance reduction due to the increment of the control and data packets traffic. In a scenario where all the nodes send messages to just a central unit, increasing the number of nodes means congestion of the nodes close to the sink and decrease the packet delivery ratio apart the routing metric under use. 


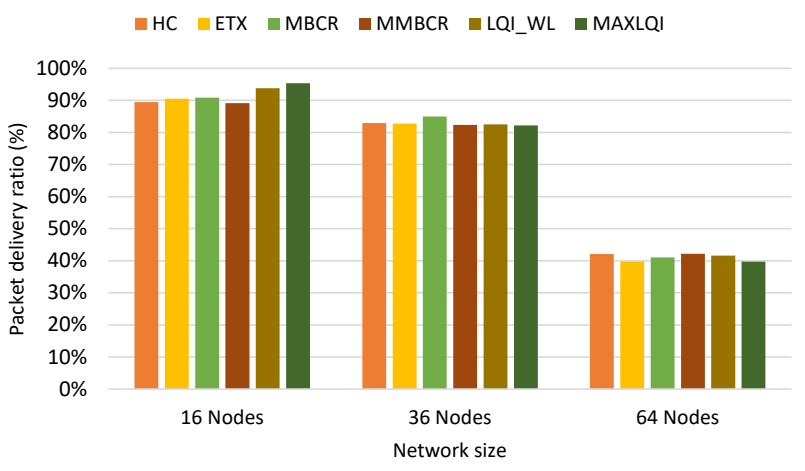

Fig. 4: Packet delivery ratio in the MP2P scenario.

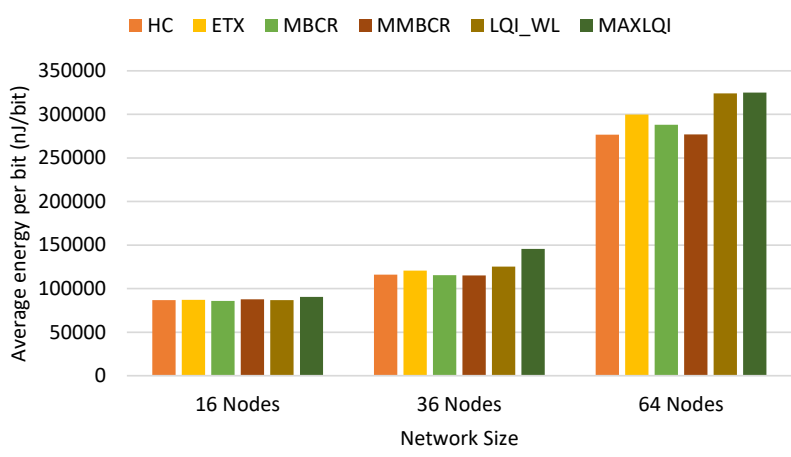

Fig. 5: Average spent energy per bit in the MP2P scenario.

Figure 5 shows the results obtained for the average spent energy per bit metric. The results present that, for all the studied metrics, the amount of energy spent to deliver data packets is lower in small networks. In the network with 64 nodes, the data packets require a higher number of forwarding messages to reach its destination thus, as consequence of the high radio usage, the consumed energy is increased. Hence, the high values of average spent energy per bit are justified due to the low packet delivery ratio (as may be seen in Figure 4) and the high power consumption. It is also possible to note that in the greater studied network, the link quality based routing metrics have the worst average spent energy per bit due to the use of routes with a big number of hops (Figure 7). Still considering the 64 nodes network, the MMBCR was able to ensure the better performance using less energy and an acceptable packet delivery ratio.

The results for the average latency metric are presented in Figure 6. As may be seen, the quantity of packets (in percentage) delivered on each latency interval (represented in $\mathrm{ms}$ ), e.g. five percent of the data messages delivered using MAXLQI have reached its destination with a latency between 100 and 150 milliseconds. For all the studied networks, the link quality based routing metrics presents the worst latency performances. The operation mode of these metrics (previously presented) seek to select routes with the best link quality without considering the distance (in hops) between nodes. This feature allows the routing protocol to forward messages through paths with a high number of hops increasing the average latency. Note that high latency is not directly related to a packet loss. Although the link quality based routing metrics have bad results for average latency, they can provide a packet delivery ratio with similar or better performance when compared with other studied routing metrics.

Figure 7 presents the results for the number of hops metric. In these figures, each color inside bars represents each number of hops value. The size of the color representation in the bar shows the quantity of packets (in percentage) delivered with that number of hops. For example, for the MBCR routing metric in the network with 16 nodes, about $60 \%$ of the packets were delivered using one hop, $30 \%$ were delivered using two hops, and $10 \%$ with three hops. The results expose that, in the networks with 16 and 36 nodes, the HC, ETX, MBCR, and MMBCR deliver more than $50 \%$ of their packets using just one hop. In contrast, the LQI_WL and MAXLQI, when compared with the other routing metrics, have presented the use of bigger paths to reach the message destination in all the studied networks. As already explained, the search for paths with better link quality can raises the use of greater paths causing high energy consumption and long average latency.

\section{B. P2P Application}

The results for the packet delivery ratio metric in the P2P scenario are available at Figure 8 . According to these results, the link quality based routing metrics have a better performance compared to the other metrics in the network with 16 and 36 nodes. However, the MAXLQI suffer a significant performance decrease with the network growth exposing its low scalability in the P2P scenario. These results show that the strategy of using just one LQI value to represent the quality of a route can fail when the paths are composed by various links. On the contrary, the LQI_WL was able to maintain a high performance even with the increment of the number of nodes in the network. The strategy to count the number of bad links allows the routing protocol to avoid the use of paths with a big number of weak links implying the packet delivery ratio increase. The results obtained by ETX are very close to the results of HC. ETX is an additive metric in function of the messages exchanged by the nodes at the MAC layer. Hence, when the information extracted from the link layer are few, ETX metric will operate just a "hop count" metric.

The results for the average energy spent per bit are exposed in Figure 9. According to the simulation experiments, in many scenarios, the link quality based routing metrics have better results, excepted in the network with 64 nodes where the MAXLQI had the lower performance compared to the other routing metrics. This worst performance of MAXLQI is a consequence of the low packet delivery ratio and the high energy consumption produced by the use of routes with a big number of hops. The energy based metrics were able to keep a low power consumption. However, its low packet delivery ratio implied high values of spent energy per delivered bit. In all studied network sizes, LQI_WL, although presenting a power consumption higher than the obtained by the energy based metrics, was able to obtain good results due to high packet delivery ratio.

Figure 10 depicts the average latency for the P2P application. The results show that, in the majority of the studied scenarios, MAXLQI has the higher average latency. Although 


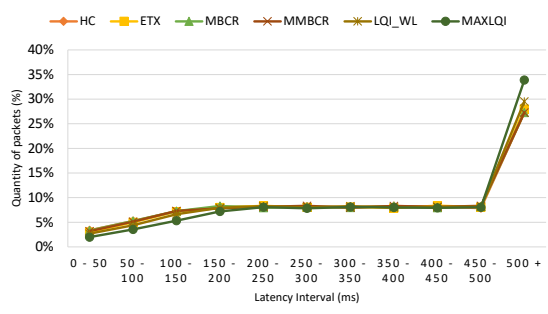

(a) 16 nodes

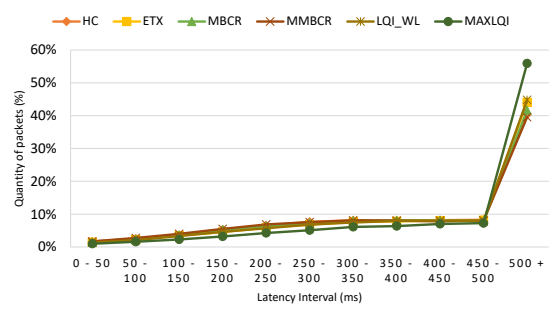

(b) 36 nodes

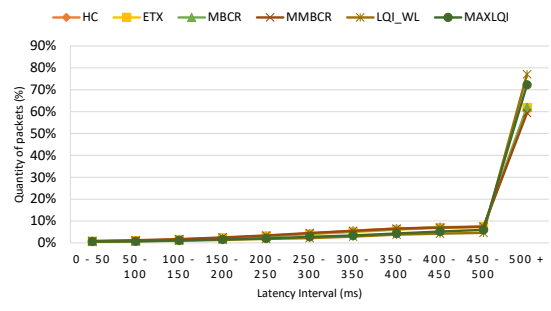

(c) 64 nodes

Fig. 6: Average latency in the MP2P scenario.

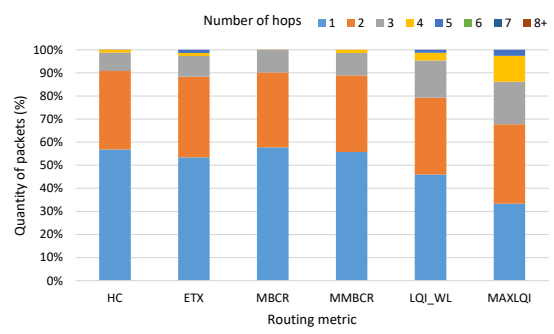

(a) 16 nodes

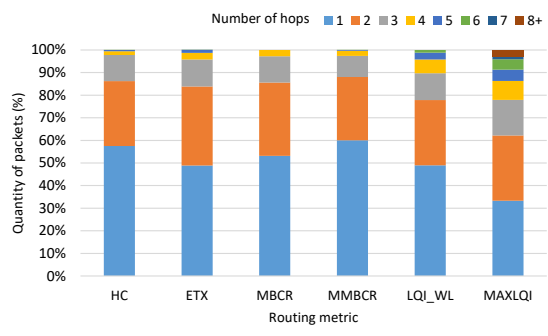

(b) 36 nodes

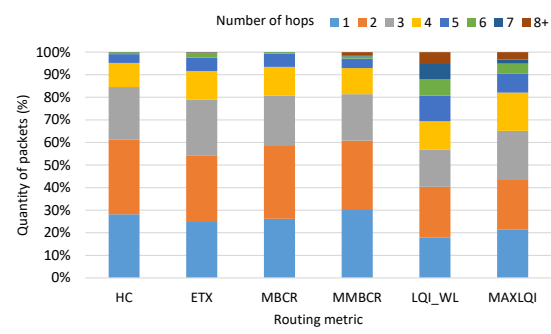

(c) 64 nodes

Fig. 7: Number of hops in the MP2P scenario.

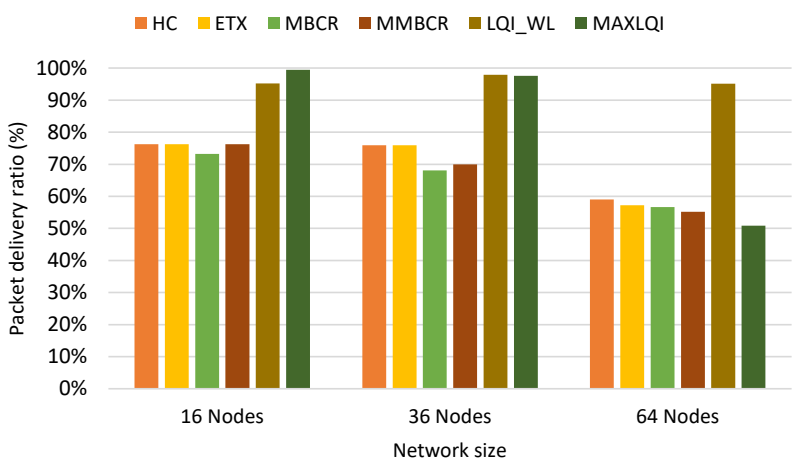

Fig. 8: Packet delivery ratio in the $\mathrm{P} 2 \mathrm{P}$ scenario.

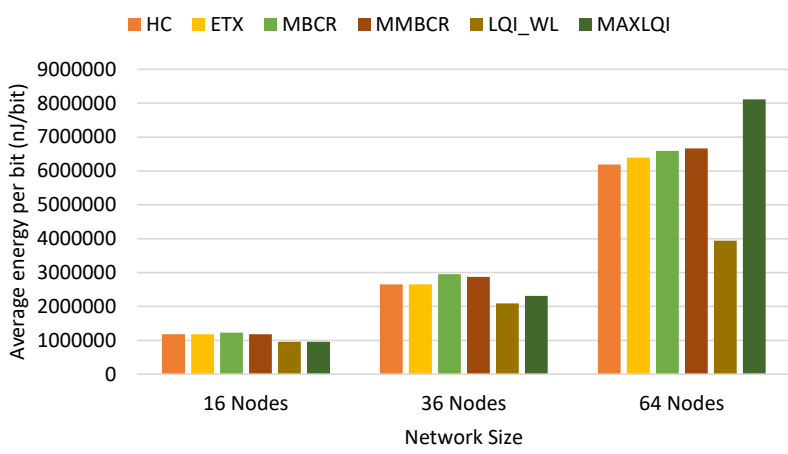

Fig. 9: Average spent energy per bit in the P2P scenario.

LQI_WL used routes with a high number of hops (presented in Figure 11), the metric stills able to achieve a latency close to the faster performed metrics. Although the number of hops may influence the average latency, this result shows that it is not the single factor that can contribute to increase the time needed to the message till reaching its destination.

The results for the number of hops metric may be seen at
Figure 11. According to the simulation results, the metrics based on link quality use routes with a high number of hops if compared with the other routing metrics, as expected. These results can directly affect the power consumption but they are not determinant for a packet delivery ratio reduction (8). Due to the distance between the sender and the receiver nodes, the number of hops used to deliver the messages is higher than in the MP2P scenario. In the network with 64 nodes, the minimum number of hops used by MAXLQI was five. In contrast, the maximum number of hops used by HC, ETX, and MBCR was four. Moreover, observing the results, it is possible to perceive that, although the network has static nodes, the distance (in hops) between the nodes may change. This effect may be caused by control messages loss at the moment of a route creation. Another justification for this behavior is the LOADng functioning. The routing protocol uses sequence numbers on its control messages that can force a node to update its routing table with a new value even the prior route being better than the current one.

\section{CONCLUSION AND FUTURE WORKS}

This work presented a performance assessment study considering several routing metrics applied to the LOADng routing protocol. LOADng is a routing protocol that has been emerged as an alternative to fulfil the drawbacks identified on the standard RPL (in LLNs). From its creation, the default version of LOADng has been studied in different scenarios and applications. However, few works are dedicated to study the impact of using different routing metrics with this protocol.

In this paper, five different routing metrics (ETX, MBCR, MMBCR, LQI_WL and MAXLQI) and the default routing metric of LOADng $(\mathrm{HC})$ were studied in network scenarios with MP2P and P2P traffic patterns. The obtained results show that link based routing metrics were able to provide 


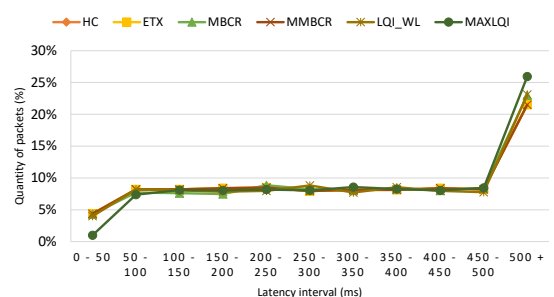

(a) 16 nodes

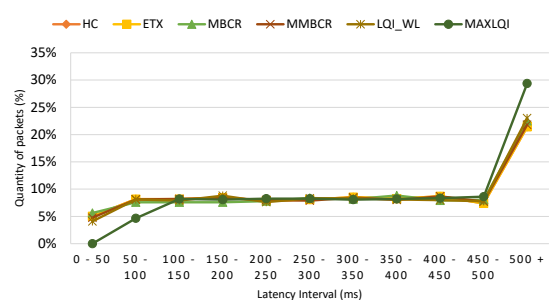

(b) 36 nodes

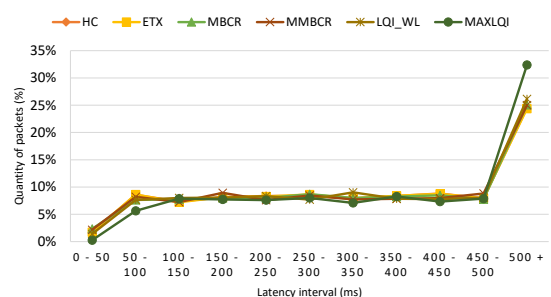

(c) 64 nodes

Fig. 10: Average latency in the $\mathrm{P} 2 \mathrm{P}$ scenario.

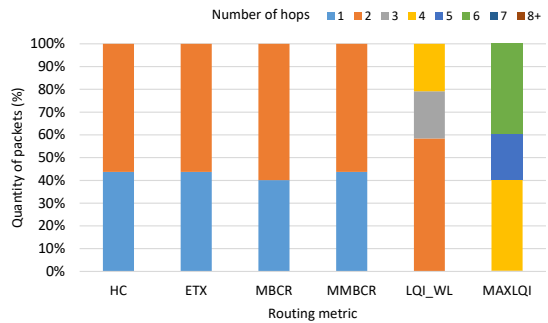

(a) 16 nodes

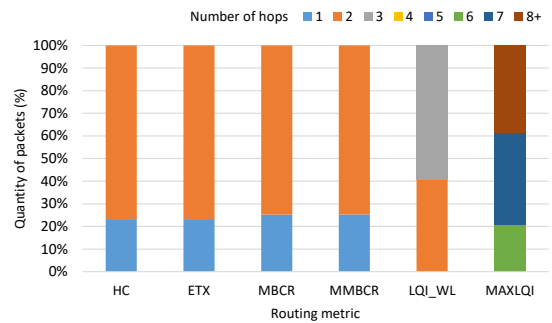

(b) 36 nodes

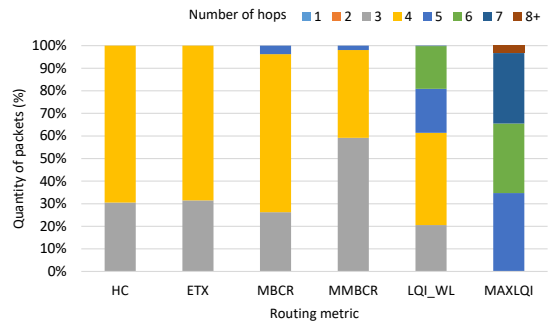

(c) 64 nodes

Fig. 11: Number of hops in the P2P scenario.

a high packet delivery ratio due to the use of most reliable paths. In contrast, these metrics have shown that, in some cases, the selection of reliable paths can use a high number of hops and cause a power consumption increase. Moreover, the use of great routes may cause a high average latency decreasing the quality of service. The ETX and the energy based routing metrics present results that, in most cases, are close to the default HC. However, the performed simulations are not enough to discard the potential of these metrics.

In an overview of the obtained results for the evaluated scenarios, LQI_WL revealed the most reliable performance when compared to the other approaches. However, the selection of a routing metric should be made considering the network requirements and the application objectives. As future work, the authors intend to extend this work considering more routing metrics and application scenarios. The authors also plan to discuss the creation of new metrics considering the ones available in the literature aiming to perform a detailed study of LOADng.

\section{REFERENCES}

[1] R. Want, B. N. Schilit, and S. Jenson, "Enabling the internet of things," Computer, vol. 48, no. 1, pp. 28-35, 2015.

[2] T. Winter, A. Brandt, J. Hui, R. Kelsy, P. Levis, K. Pister, R. Struik, J. Vasseur, and R. Alexander, "Rpl: Ipv6 routing protocol for low-power and lossy networks," Internet Requests for Comments, RFC Editor, RFC 6550, 2012.

[3] O. Iova, G. P. Picco, T. Istomin, and C. Kiraly, "Rpl, the routing standard for the internet of things... or is it?" IEEE Communications Magazine, vol. 17, 2016.

[4] C. H. Barriquello, G. W. Denardin, and A. Campos, "A geographic routing approach for ipv6 in large-scale low-power and lossy networks," Computers \& Electrical Engineering, 2015.

[5] H. Fotouhi, D. Moreira, and M. Alves, "Mrpl: Boosting mobility in the internet of things," Ad Hoc Networks, vol. 26, pp. 17-35, 2015.

[6] T. Clausen, J. Yi, A. Niktash, Y. Igarashi, H. Satoh, U. Herberg, C. Lavenu, T. Lys, and J. Dean, "The lightweight on-demand ad hoc distance-vector routing protocol-next generation (loadng)," Working
Draft, IETF Secretariat, Internet-Draft draft-clausen-lln-loadng-14.txt, 2016.

[7] C. Perkins, E. Belding-Royer, and S. Das, "Ad hoc on-demand distance vector (aodv) routing," Internet Requests for Comments, RFC Editor, RFC 3561, 2003.

[8] M. Vučinić, B. Tourancheau, and A. Duda, "Performance comparison of the rpl and loadng routing protocols in a home automation scenario," in 2013 IEEE Wireless Communications and Networking Conference (WCNC). IEEE, 2013, pp. 1974-1979.

[9] J. Tripathi, J. C. De Oliveira, and J.-P. Vasseur, "Proactive versus reactive routing in low power and lossy networks: Performance analysis and scalability improvements," Ad Hoc Networks, vol. 23, pp. 121-144, 2014.

[10] J. Yi, T. Clausen, and Y. Igarashi, "Evaluation of routing protocol for low power and lossy networks: Loadng and rpl," in 2013 IEEE Conference on Wireless Sensor (ICWISE). IEEE, 2013, pp. 19-24.

[11] S. Elyengui, R. Bouhouchi, and T. Ezzedine, "Loadng routing protocol evaluation for bidirectional data flow in ami mesh networks," Int. Journal of Emerging Technology and Advanced Engineering, 2015.

[12] J. V. Sobral, J. J. Rodrigues, K. Saleem, and J. Al-Muhtadi, "Performance evaluation of loadng routing protocol in iot $\mathrm{p} 2 \mathrm{p}$ and $\mathrm{mp} 2 \mathrm{p}$ applications," in International Multidisciplinary Conference on Computer and Energy Science (SpliTech). IEEE, 2016, pp. 1-6.

[13] P. Karkazis, P. Trakadas, H. C. Leligou, L. Sarakis, I. Papaefstathiou, and T. Zahariadis, "Evaluating routing metric composition approaches for qos differentiation in low power and lossy networks," Wireless networks, vol. 19, no. 6, pp. 1269-1284, 2013.

[14] T. Clausen, J. Yi, C. Lavenu, A. Lys, A. Niktash, Y. Igarashi, and H. Satoh, "The lln on-demand ad hoc distance-vector routing protocolnext generation (loadng)," Working Draft, IETF Secretariat, InternetDraft draft-clausen-1ln-loadng-00.txt, 2011.

[15] J.-P. Vasseur, M. Kim, K. Pister, N. Dejean, and D. Barthel, "Routing metrics used for path calculation in low-power and lossy networks," Internet Requests for Comments, RFC Editor, RFC 6551, 2012.

[16] A. Brachman, "Rpl objective function impact on llns topology and performance," in Internet of Things, Smart Spaces, and Next Generation Networking. Springer, 2013, pp. 340-351.

[17] Y. Yang, J. Wang, and R. Kravets, "Designing routing metrics for mesh networks," in IEEE Workshop on Wireless Mesh Networks (WiMesh), 2005.

[18] C.-K. Toh, "Maximum battery life routing to support ubiquitous mobile computing in wireless ad hoc networks," IEEE Communications Magazine, vol. 39, no. 6, pp. 138-147, 2001.

[19] C. Gomez, A. Boix, and J. Paradells, "Impact of lqi-based routing metrics on the performance of a one-to-one routing protocol for ieee 802.15. 
4 multihop networks," EURASIP Journal on Wireless Communications and Networking, vol. 2010, p. 6, 2010.

[20] D. S. De Couto, D. Aguayo, J. Bicket, and R. Morris, "A high-throughput path metric for multi-hop wireless routing," Wireless Networks, vol. 11, no. 4, pp. 419-434, 2005.

[21] A. Boulis et al., "Castalia: A simulator for wireless sensor networks and body area networks," NICTA: National ICT Australia, 2011.

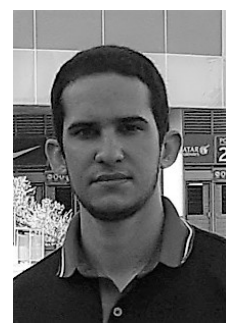

José V. V. Sobral (jose.sobral@it.ubi.pt) is currently a Ph.D. student at the University of Beira Interior (UBI), Covilhã, Portugal. He received his M.Sc. degree in Computer Science from the Federal University of Piauí (UFPI), Teresina, Brazil, and B.S. degree in Computer Science from the Centro de Ensino Unificado de Teresina (CEUT), Teresina, Brazil. José Sobral is an assistant professor at the Federal Institute of Maranhão (IFMA), São Luís, Brazil, and a member of NetGNA Research Group. His research interests include Internet of Things (IoT), routing protocols for low power and lossy networks, wireless sensors networks, RFID systems, and computational intelligence.

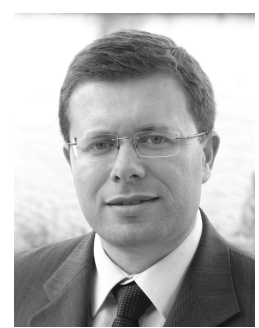

Joel J.P.C. Rodrigues (joeljr@ieee.org) [S’01, M'06, SM'06] is a professor and senior researcher at the National Institute of Telecommunications (Inatel), Brazil and senior researcher at the Instituto de Telecomunicaes, Portugal. He has been professor at the University of Beira Interior (UBI), Portugal and visiting professor at the University of Fortaleza (UNIFOR), Brazil. He received the Academic Title of Aggregated Professor in informatics engineering from UBI, the Habilitation in computer science and engineering from the University of Haute Alsace, France, a $\mathrm{PhD}$ degree in informatics engineering and an MSc degree from the UBI, and a five-year BSc degree (licentiate) in informatics engineering from the University of Coimbra, Portugal. His main research interests include e-health, sensor networks and IoT, vehicular communications, and mobile and ubiquitous computing. Prof. Joel is the leader of NetGNA Research Group (http://netgna.it.ubi.pt), the President of the scientific council at ParkUrbis Covilh Science and Technology Park, the Past-Chair of the IEEE ComSoc Technical Committee on eHealth, the Past-chair of the IEEE ComSoc Technical Committee on Communications Software, Steering Committee member of the IEEE Life Sciences Technical Community and Publications co-Chair, and Member Representative of the IEEE Communications Society on the IEEE Biometrics Council. He is the editor-in-chief of the International Journal on E-Health and Medical Communications, the editor-in-chief of the Recent Advances on Communications and Networking Technology, the editor-in-chief of the Journal of Multimedia Information Systems, and editorial board member of several high-reputed journals. He has been general chair and TPC Chair of many international conferences, including IEEE ICC, GLOBECOM, and HEALTHCOM. He is a member of many international TPCs and participated in several international conferences organization. He has authored or coauthored over 500 papers in refereed international journals and conferences, 3 books, and 2 patents. He had been awarded several Outstanding Leadership and Outstanding Service Awards by IEEE Communications Society and several best papers awards. Prof. Rodrigues is a licensed professional engineer (as senior member), member of the Internet Society, an IARIA fellow, and a senior member ACM and IEEE.

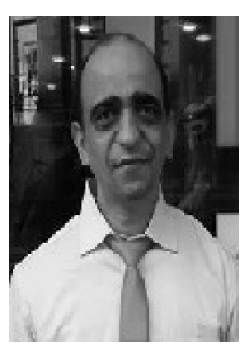

Neeraj Kumar (neeraj.kumar@thapar.edu) received his Ph.D. in CSE from SMVD University, Katra (J \& $\mathrm{K})$, India, and was a postdoctoral research fellow in Coventry University, Coventry, UK. He is working as an Associate Professor in the Department of Computer Science and Engineering, Thapar University, Patiala (Pb.), India since 2014. Dr. Neeraj is an internationally renowned researcher in the areas of VANET \& CPS Smart Grid \& IoT Mobile Cloud computing \& Big Data and Cryptography. He has published more than 150 technical research papers in leading journals and conferences from IEEE, Elsevier, Springer, John Wiley. His paper has been published in some of the high impact factors journals such as-IEEE Transactions on Industrial Informatics, IEEE Transactions on Industrial Electronics, IEEE Transactions on Information Forensics and Security, IEEE Transactions on Dependable and Secure Computing, IEEE Transactions on Power Systems, IEEE Transactions on Vehicular Technology, IEEE Systems Journal, IEEE Wireless Communication Magazine, IEEE Vehicular Technology Magazine, IEEE Communication Magazine, IEEE Networks Magazine etc. Apart from the journals conferences, he has also published papers in some of the core conferences of his area of specialization such asIEEE Globecom, IEEE ICC, IEEE Greencom, IEEE CSCWD. He has guided many research scholars leading to Ph.D. and M.E./M.Tech. His research is supported by funding from TCS, CSIT, UGC and UGC in the area of Smart grid, energy management, VANETs, and Cloud computing. He is member of the cyber-physical systems and security research group. He has research funding from DST, CSIR, UGC, and TCS. He has total research funding from these agencies of more than 2 crores. He has got International research project under Indo-Poland and Indo-Austria joint research collaborations in which teams from both the countries will visit to Thapar University, Patiala and Warsaw University, Poland, University of Innsburg, Austria respectively. He has h-index of 25 (according to Google scholar, March 2017) with 2500 citations to his credit. He is editorial board members of International Journal of Communication Systems, Wiley, and Journal of Networks and Computer Applications, Elsevier. He has visited many countries mainly for the academic purposes. He is a visiting research fellow at Coventry University, Coventry, UK. He has many research collaboration with premier institutions in India and different universities across the globe. He is a member of IEEE.

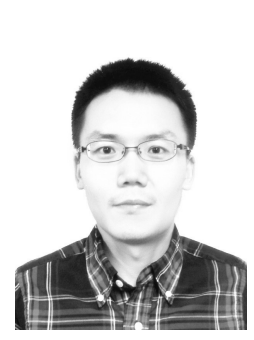

Chunsheng Zhu (chunsheng.tom.zhu@gmail.com) received the Ph.D. degree in Electrical and Computer Engineering from The University of British Columbia, Canada. He is currently a Post-Doctoral Research Fellow with the Department of Electrical and Computer Engineering, The University of British Columbia, Canada. He has authored over 100 papers published or accepted by refereed international journals/magazines (e.g., IEEE Transactions on Industrial Electronics, IEEE Transactions on Computers, IEEE Transactions on Information Forensics and Security, IEEE Transactions on Emerging Topics in Computing, IEEE Systems Journal, IEEE Access, and IEEE Communications Magazine) and conferences (e.g., IEEE Globecom and IEEE ICC). His current research interests mainly include wireless sensor networks, cloud computing, Internet of Things, social networks, and security.

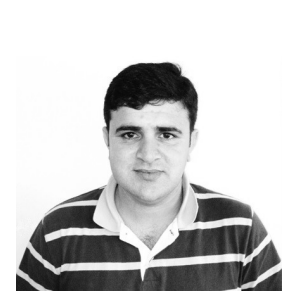

Raja W. Ahmad (wasimraja@ciit.net.pk) is an Assistant professor at COMSATS Institute of Information Technology, Pakistan. He did his $\mathrm{PhD}$ in Computer Science from University of Malaya under Bright Spark Scholarship program. He started his carrier as a computer student back to 2003 by choosing computer science as major during undergraduation course from University of Azad Jammu \& Kashmir, Muzaffarabad. In addition, he did his masters from COMSATS Institute of Information Technology, Abbottabad, Pakistan under "COMSATS Merit Scholarship" program. His research interests include, mobile application energy profiling, energy efficient computational offloading, cloud resource allocation, VM migration, Network performance, Application's QoS on low bandwidth networks, and energy efficient cloud data centers. 